\title{
o Perquín: fiesta y desarrollo territorial ${ }^{1}$
}

\author{
Fernando Villatoro \\ Universidad Centroamericana "José Simeón \\ Cañas" (El Salvador)
}

Resumen: El Festival de Invierno de Perquín (FIP) desarrollado desde hace 25 años en el Departamento de Morazán en El Salvador busca preservar la memoria histórica salvadoreña a través de diferentes actividades culturales que recuerdan lo vivido durante el conflicto armado salvadoreño. Perquín se ha desarrollado territorialmente estableciendo relaciones entre la cultural local, el sistema social de actores y el entramado político-institucional del municipio, así como las diferentes instituciones del Estado que confluyen en el territorio. Estas relaciones entre lo local y lo nacional se hacen explícitas en el festival y han logrado desarrollar una marca-destino que contribuye a generar un desarrollo territorial sistémico y sostenible.

Palabras clave: festival, Perquín, desarrollo, sistema, territorio.

Abstract: The Winter Festival Perquin (FIP) developed for 25 years in the Department of Morazán in El Salvador seeks to preserve the historical memory salvadoreña through different cultural activities that remember what lived during the Salvadoran armed conflict. Perquin has been developing territorially establishing relationships between local cultural, social system actors and political-institutional framework of the municipality as well as the different state institutions that meet in the territory. These relationships between the local and the national are made explicit in the festival and have developed a brand destination that contributes to a systemic and sustainable territorial development.

Keywords: festival, Perquin, development, system, territory. 


\section{Antecedente}

Mi relación con el departamento de Morazán empezó en los años setenta, mucho antes de que yo naciera; casi cinco años antes de los desplazamientos de miles de personas hacia los antiguos campamentos de refugiados en Honduras, y es que mi nombre lo debo al cariño que mi padre le tomó al primer lugar donde empezó a trabajar: San Fernando, Morazán.

De camino al municipio, gracias al cual tengo mi nombre, se encuentra un pequeño poblado que ha sido protagonista de la historia salvadoreña y que lleva 25 años desarrollando un festival, desde el cual se busca rescatar la memoria histórica de todo un país. Me refiero a Perquín, el cual forma parte del territorio "Donde el viento te susurra historias".

Perquín se encuentra a 204 km de San Salvador, la ciudad capital de El Salvador. El camino que te lleva al municipio refleja la belleza de un pequeño país, el cual se puede transitar en vehículo en un par de horas; pero si se trata de recorrer su historia reciente, hacen falta muchísimas horas de conversaciones, vivencias y diálogos inconclusos de un pueblo que ha venido construyendo sus sueños desde hace más de dos décadas. Es por eso que a medida te acercas al lugar, las historias surgen: todos tienen una historia que contar.

En la década de los ochenta, el conflicto armado entre el Ejército salvadoreño y los grupos guerrilleros obligó a miles de personas a refugiarse fuera de las fronteras nacionales, constituyendo comunidades en las que a juicio de Artiga-González (1993), se vivieron procesos solidarios y de cohesión y organización social entre refugiados.

Muchas familias aún recuerdan lo vivido en el campamento de Colomoncagua, la vida cotidiana de las comunidades en el exilio, quiénes eran los referentes sociales $y$, sobre todo, cómo construyeron un nuevo orden social a pesar de estar en un contexto marcado por la hostilidad del gobierno salvadoreño de la época y por las fuerzas armadas hondureñas. En ese tiempo, los cercos militares, la sospecha y la presión psicológica sobre las poblaciones era lo cotidiano para la comunidad.

Ahora, después de 24 años de la firma de los Acuerdos de Paz entre las partes en conflicto y a casi 26 años de que los campamentos de Mesa Grande, San Antonio y Colomoncagua fueran repatriados hacia El Salvador y se instalaran en diferentes comunidades de Morazán, el recuerdo aún sigue vivo. En esa 
época, a finales de los ochenta y al volver a las comunidades de origen, era necesario reconstruir lo que años atrás era su hogar así como el debilitado sistema social existente. En este contexto nació el Festival de Invierno de Perquín (FIP), con el fin de transmitir un sentimiento de reunificación entre las comunidades locales.

Cuando terminó la guerra, los guerrilleros que estaban cumpliendo el cese de fuego y la población civil que se mantuvo viviendo en la zona durante los años del conflicto acordaron celebrar el primer festival, esto con el objetivo de transmitir a los salvadoreños que en Morazán la guerra había terminado y que deseaban reunificarse con el resto de la población (Perquín, 2015). De esta manera, la fiesta local se convertiría en una especie de mecanismo de mediación en la re-construcción del sistema social y el desarrollo de la cultura (Cuenca Cabeza, 2000).

El festival se desarrolla entre el 2 y 6 de agosto, y actualmente atrae entre 20 y 25 mil turistas según Prodetur, quien es la encargada de promover el turismo en la zona norte de Morazán. Uno de los fines del festival es promover la historia, la cultura y la naturaleza del municipio, dando prioridad a grupos locales de danza folclórica y música autóctona. También se fomenta la producción artesanal, las tradiciones locales, las exposiciones sobre la memoria histórica de la zona y una caminata a El Mozote, sitio histórico donde se rinde homenaje a las víctimas de la masacre sucedida en 1981 (La Prensa Gráfica, 2014).

Por su parte, la ex alcaldesa del municipio, Mirian Rodríguez, menciona que el festival es un espacio donde se puede recordar la historia de los últimos años del país y, principalmente, la historia de la zona. A través de las actividades culturales realizadas en Perquín, los habitantes han desarrollado lo establecido por la Conferencia Internacional de la UNESCO en 1982 y han entendido la cultura como:

El conjunto de los rasgos distintivos, espirituales y materiales, intelectuales y afectivos que caracterizan a una sociedad o un grupo social. Ella engloba, además de las artes y las letras, los modos de vida, los derechos fundamentales al ser humano, los sistemas de valores, las tradiciones y las creencias (UNESCO, 1982, pp. 1).

Cuenca Cabeza (2000) plantea que esta declaración es una visión aceptada por el conjunto de gobernantes miembros de la UNESCO, 
existiendo así un consenso entre las distintas tendencias ideológicas. El Festival de Invierno de Perquín ha pasado de pequeñas actividades recreativas a convertirse en una pequeña Industria Cultural Creativa (ICC), de acuerdo a las condiciones de El Salvador y entendido éste concepto como: "aquellos sectores de actividad organizada que tienen como objeto principal la producción, reproducción, promoción, difusión y/o la comercialización de bienes, servicios y actividades de contenido cultural, artístico o patrimonial" (UNESCO, 2015).

\section{Estructuras}

El desarrollo territorial se refiere al proceso de transformación continua por el que diversas estructuras interactúan de forma sistémica entre sí. Mientras que la sostenibilidad significa garantizar las necesidades de la generación presente sin comprometer la capacidad de las generaciones futuras para satisfacer sus propias necesidades (Comisión Mundial del Medio Ambiente y del Desarrollo, 1992, pp. 29). Por lo anterior, en el presente artículo quiero plantear el término Desarrollo Territorial sistémico y sostenible con el fin de responder a los procesos complejos que se dan en el territorio.

En el informe titulado Nuestro futuro común, la Comisión Mundial del Medio Ambiente y del Desarrollo (WCED, por sus siglas en inglés) establece que el desarrollo sostenible está separado en tres pilares, los cuales buscan transformar o proteger de manera equilibrada las dimensiones 0 subsistemas: económico, social y medioambiental. Sin embargo, recientemente, hoy la cultura es un cuarto pilar de desarrollo sostenible (Hawkes, 2001). Cada uno de estos pilares o subsistemas, así como su interrelación permite el desarrollo sistémico y sostenible, en tanto que todo sistema está compuesto por subsistemas integrados y organizados entre sí y con diferentes estructuras (Bertalanffy, 1987).

Boisier (2011) plantea que un territorio es un espacio ocupado por comunidades o sociedades estables, con un patrón de asentamiento humano discernible; con estructuras sociales, tecnológicas y económicas; con cultura e identidad. Es precisamente el lugar donde el ser humano nace, se educa, trabaja, forma su familia, desarrolla servicios varios y probablemente donde será sepultado. La relación de las personas con el territorio es tal que si el territorio tiene un desarrollo progresivo sus habitantes tendrán más probabilidades de acceder a buenas condiciones de vida. En cambio, si el territorio sufre deterioro sus habitantes padecerán las consecuencias. 
Un enfoque sistémico permitirá comprender y explicar los procesos que se dan en el territorio. Es importante prestar atención a los vínculos entre elementos y revisar cuáles son los objetivos compartidos que hacen que el sistema interactúe con el entorno. Este planteamiento advierte sobre la importancia de la interdependencia de los elementos que configuran un sistema y de éste con el entorno. La interdependencia es un factor clave para orientar la acción pública y privada en un sistema territorial y puede extender y optimizar las capacidades existentes o contraerlas.

Por otra parte, la organización interna de un sistema se expresa en su estructura, es decir, en la manera cómo se han ido definiendo y aplicando los vínculos entre todos los elementos que lo conforman. La estructura ejerce un rol fundamental, ya que le corresponde el ordenamiento y la fluidez de los vínculos entre los elementos del sistema.

La estructura está limitada por dos puntos críticos: el primero de ellos, de signo negativo, expresa el comienzo de la destrucción del sistema cuando la entropía, o desorden entre los vínculos de los elementos, obstruye el procesamiento y distribución de la energía o los insumos que el sistema recibe de su entorno. El segundo de ellos, el positivo, cuando los canales o vínculos permiten el procesamiento y distribución sinérgica de la energía, provocando que el sistema alcance su funcionamiento óptimo $y$, por ende, tienda al equilibrio interno y con el entorno.

Tal parece que el festival, al nacer junto al nuevo sistema social del territorio, ha logrado generar interdependencia entre sus elementos y no ha tenido que luchar contra la estructura territorial, sino todo lo contrario, ha sabido procesar y distribuir la energía que obtiene del entorno. Los elementos del subsistema cultural como festivales, artesanías y actividades de ocio de la localidad, reciben energía del entorno territorial a través de la cohesión social, la educación (elementos del subsistema social) $y$ de instituciones (elementos del subsistema institucional). Lo anterior ha permitido que el festival siga vigente después de tantos años y que haya evolucionado de la manera que lo ha hecho.

Las actividades pautadas en la agenda cultural del territorio, en el caso del festival de Perquín, surgen de las propuestas de los grupos locales que se apoyan en diversas instituciones del territorio como la Casa de la Cultura, escuelas, centros de formación, comités turísticos, comité de festejos municipales, artesanos locales y regionales, compañías musicales locales, Comisión Nacional de la Micro y Pequeña Empresa (CONAMYPE), 
entre otras. Lo anterior demuestra el ordenamiento y la fluidez de los vínculos entre los elementos del sistema que la estructura territorial ha creado.

\section{Destino}

El festival nació con el objetivo recordar la memoria histórica, con el fin de preservar la cohesión social que impulsó el nuevo orden social en tiempos de paz. Es innegable que un evento como éste ahora se ha convertido en una especie de "marca destino", lo cual para Fernández Cavia y Huertas (2014) está centrado en la actividad turística y se ocupa principalmente en atraer visitantes hacia un destino turístico.

Ritchie (1998) plantea que la marca de destino puede desempeñar un papel de coordinación, pero también necesita que otros públicos se pongan de acuerdo con ésta, ya que sólo es una parte de todo el esfuerzo ese proceso de creación, implementación y desarrollo de marca territorial (Fernández Cavia, 2013). También genera desarrollo turístico, identidad local, amor por el territorio,actividades de ocio,ingresos locales, empleos, entre otros. Se puede inferir que si el festival logra mejorar la calidad de vida, entonces estará generando desarrollo humano para los habitantes del territorio.

Al evolucionar de un simple festival a una referencia de destino, es claro que las interrelaciones, parte fundamental para el funcionamiento de cualquier sistema, han funcionado en el caso de Perquín. Sin embargo, para que existan interrelaciones deben existir enlaces entre las partes y comprender cuáles de esos enlaces ayudan a entender si existen interrelaciones e incluso a determinar el tipo de interrelaciones a partir de la clase de enlace.

Los enlaces no se deben entender como una relación lineal, sino como una red que genera procesos circulares, es decir, que no tienen principio ni fin. Los cambios en los elementos de los subsistemas, a partir de las interrelaciones, determinan la calidad del sistema territorial. Estos cambios incrementan en la medida que aumenta su complejidad y se vuelven impredecibles según las características individuales de los actores o colectivos que componen el sistema territorial.

Los enlaces del sistema territorial de Perquín están marcados por la memoria histórica; por las historias que las familias cuentan a sus miembros más jóvenes y por las representaciones simbólicas que se generan al interior del territorio. Estos enlaces determinan las 
interrelaciones entre artesanos, muralistas y bailarines con las ONG, la alcaldía, la gobernación departamental, las asociaciones comunales y las escuelas. Los enlaces en Perquín han propiciado que cada año aumenten las interrelaciones entre los diversos elementos de los subsistemas, por lo tanto, el fortalecimiento de estas interrelaciones ha permitido que el festival se siga desarrollando durante 25 años de forma consecutiva y se estima la llegada de casi 25,000 turistas, toda una industria si tomamos en cuenta el tamaño de la población y las características del municipio.

\section{Territorio}

El territorio se determina a partir de estructuras que contienen elementos y que se relacionan para determinar lo que sucede en el mismo. Dichas relaciones son las que permiten que el territorio como sistema tenga la habilidad de adaptarse a los cambios del entorno y garantizar su sostenibilidad. También existe una relación entre sistema y entorno que permite que uno influya en el otro y viceversa (Tobey, 2007), por eso es necesario diferenciar entre un sistema y su entorno, estableciendo una frontera (aunque sea de manera subjetiva) que ayude a determinar qué está dentro o fuera del sistema (Johansen, 1993).

Por su parte, Luhmann (1998) establece la diferencia entre sistema y entorno con una línea divisoria que permite establecer la relación existente entre ellos, además reconoce que el sistema se determina a partir del objeto de análisis y que, por tanto, el entorno se presenta a partir del punto de vista del sistema.
Vergara (2012) propone entender el territorio como la construcción de un proyecto-trayecto que no está dado sin más, sino que es construido, creado imaginativamente. Este imaginario tiende a ser materializado según las circunstancias históricas y sociales, como en el caso de Perquín. En este sentido, el territorio es parte de un sueño colectivo en el que han de concretarse sus fines y valores porque cree en ellos.

Un planteamiento similar es el de Belkis (2007), quien propone que la vocación del territorio está determinada por los recursos, las potencialidades, nivel de desarrollo, visión compartida de sus habitantes, modo de vida de las personas que lo habitan, cultura local, historia de sus instituciones y el papel que juega en el ámbito regional, nacional e internacional. Tomar en cuenta lo anterior servirá de base para el ordenamiento territorial y para la promoción económica y social que realicen los diversos actores. Se trata de que toda actividad cultural retome 
los elementos del tejido social, que se apoye en organizaciones e instituciones locales, que planifique y financie actividades culturales. También es necesario potenciar la creación de nueva infraestructura local, de medios de comunicación que promueven las actividades de competencias y saberes locales.

\section{Proceso}

Volviendo al marco del Festival de Invierno,es decir, los bailes folclóricos, las artesanías hechas con materiales locales, las caminatas, las actividades de ocio, entre otras. Me atrevería a decir que el festival ha evolucionado de pequeñas actividades recreativas a generar una pequeña industria cultural y creativa. Es de recordar que los territorios también tienden a sufrir procesos entrópicos como efecto del crecimiento poblacional, la convivencia social, las políticas nacionales, la globalización o los cambios de modelos económicos. En casos como estos, la cultura, a partir de sus diferentes industrias culturales y creativas, sirve para obtener energía de su entorno y organiza el territorio de forma que éste pueda conseguir el equilibrio.

Para entender mejor los efectos de las ICC sobre el desarrollo territorial sistémico y sostenible es preciso analizarlo a partir de los planteamientos de autores como Bertalanffy, Johansen, Boulding, Luhmann, Easton entre otros. Dichos autores señalan que las características más comunes de un sistema están determinadas por la energía que éste obtiene de su entorno, por el proceso de conversión, los productos del sistema y la retroalimentación que recibe.

En nuestro análisis tomaremos las características planteadas por Johansen, asumiendo que dichos conceptos varían con lo expuesto por otros autores, pero que en esencia se refieren al mismo proceso circular y a la relación entre el sistema y su entorno.

Dicho proceso tienen como parte:

a) Corrientes de entrada: son los recursos que el sistema importa de su entorno para poder funcionar; b) Conversión de los recursos: es el modo de organizar el sistema y alcanzar sus objetivos; c) Corrientes de salida: son los recursos que el sistema devuelve a su entorno y d) Retroalimentación: es un mecanismo de control, cuya finalidad es corregir el proceso de conversión de recursos.

Los recursos humanos, tecnológicos, económicos, las instituciones y los medios de comunicación son corrientes de entrada que, posteriormente, los diversos 
subsistemas convertirán con el fin de organizarlos y alcanzar sus objetivos, preservación histórica, atracción turística, generación de ingresos, educación, ocio y cohesión social.

En este dinamismo, los recursos convertidos emergen del sistema en forma de organizaciones que trabajan juntas y con mayor participación ciudadana para tomar la forma de imaginarios colectivos, historias vividas, infraestructura turística, desarrollo económico, fortalecimiento institucional, entre otros, representando así corrientes de salida que, a través de un proceso de retroalimentación, vuelven a ingresar al sistema para afrontar objetivos cada vez más complejos.

Si la retroalimentación o las corrientes de entrada no proveen recursos suficientes para organizar y alcanzar los objetivos del sistema, los procesos de conversión serán deficientes y los recursos emergentes del sistema no serán suficientes para generar desarrollo sostenible, ya que la entropía será mayor que la entropía negativa dentro del sistema.

En un escenario de no desarrollo $y$ de deterioro del sistema, los productos del sistema serán organizaciones descoordinadas, con poca participación ciudadana, desintegración social, falta de infraestructura social, debilitamiento institucional, degradación de medio ambiente e incluso incremento en los índices de migración.

Las Industrias Culturales Creativas sirven como recursos de entrada que ayudan a organizar el sistema y alcanzar sus objetivos, por tanto, los recursos que saldrán del sistema estarán contribuyendo a generar procesos de desarrollo sostenible.

Dicho desarrollo sostenible debe fomentar las industrias culturales nacionales (Quartesan, Romis, y Lanzafame, 2007) a través de nuevos espacios culturales que generen competitividad y respondan estratégicamente a iniciativas locales y que estimulen los procesos de desarrollo endógeno (Vázquez Barquero, 2000).

La cultura y los valores locales determinan los procesos de desarrollo territorial y se convierten en una condición necesaria para la consolidación de procesos de desarrollo cultural. También estas industrias culturales generan un sentimiento de pertenencia a una comunidad local. Este sentimiento fuertemente arraigado se sobrepone al conflicto de clases e incluso puede llegar a modificar las relaciones laborales y limitar los conflictos sociales (Vázquez Barquero, 2000).

Para Martín Baró (1995) "la conciencia de pertenecer a uno u 
otro grupo -comunidad local afecta lo que las personas son y hacen". La identidad territorial generada por las ICC tiene una base objetiva de elementos compartidos por una población, más una conciencia sobre las características comunes que movilizan a las personas como grupo social (Martín-Baró, Blanco y Chomsky, 1998). Arocena (2001) plantea que en las comunidades identitarias se encuentran: a) el proyecto territorial $u$ objetivos territoriales, b) la integración social y c) la cultura de la información.

Sobre el proyecto territorial, Arocena (2001) asegura que los procesos de identidad colectiva deben articular el pasado, con el presente de los objetivos territoriales, ya que cuando la identidad queda en la nostalgia de un pasado mejor, se convierte en un obstáculo al desarrollo. Mientras que en la integración social, la identidad es la palanca del desarrollo al ser capaz de generar objetivos colectivos. Por su parte, la cultura de la información aumenta el nivel de conocimiento e información generada localmente.

Las ICC y el desarrollo territorial sistémico y sostenible fortalecen las identidades locales, la creatividad y la formación de capital social (Boisier, 2005) aprovechando sus saberes, sus tradiciones, su identificación con el territorio y agregando valor a su actividades culturales para integrarse de mejor manera al entorno territorial.

\section{Conclusiones}

Las ICC influyen en el desarrollo territorial sistémico y sostenible estableciendo sentimientos de pertenencia. Estos sentimientos también se ven reforzados por el idioma, la comida, la historia, personajes famosos, equipos de fútbol, sentimientos nacionalistas y otros. Es necesario que los territorios cuenten con datos que le permitan identificar los enlaces que se van creando a lo largo del tiempo. Los enlaces se pueden identificar a través de las encuestas de opinión, observación de los comportamientos, a través de los habitantes, estudios sobre consumos culturales, uso del tiempo libre e itinerarios de ocio.

Una vez determinados los enlaces se deben analizar las interrelaciones que se dan al interior del sistema territorial. Éstas se determinan a partir del número de asociaciones culturales existentes, frecuencias en las reuniones, el trabajo que realizan con otras instituciones afines y no afines a ellas, el perfil de los miembros, las formas de integrar a nuevos miembros o nuevos actores y la capacidad de trabajo en equipo. 
Las interrelaciones que fomenten la cooperación entre los diferentes elementos de los subsistemas se deben preservar,ya que garantizan un trabajo armonioso entre los actores. Mientras que en las interrelaciones que se consideren destructivas se debe cortar los enlaces que la alimentan ya que podrían afectar al sistema territorial.

Las instituciones locales que definen el objetivo de lograr la cohesión social deberán fomentar las interrelaciones entre las diferentes propuestas de actividades culturales, esto con el fin de incluir grupos sociales externos al territorio: minorías étnicas, mejorar la convivencia en las comunidades, trabajar en los imaginarios colectivos. Todo esto con el fin de mejorar el grado de participación social y la construcción de identidad local (UNESCO, 2014).

El nacimiento del Festival de Invierno en Perquín junto con las actividades vinculadas a él, surgen con el fin de restaurar el orden social en los nuevos tiempos de paz y para hacer una promoción turística del territorio. Muchas de las representaciones culturales aparecen debido a la necesidad de expresar algún sentimiento individual o colectivo.

El desarrollo territorial sistémico y sostenible está integrado por un tejido ilimitado de elementos que se interrelacionan entre sí y que generan influencia recíproca dentro de un mismo sistema territorial o desde el sistema hacia su entorno produciendo cambios en el sistema.

Las industrias culturales y creativas son elementos que surgen de un territorio con el fin de generar cambios al interior del mismo territorio. Por tanto, se puede concluir que las actividades culturales se apoyan en elementos del entorno tomando su energía para cambiar el sistema local, y una vez que ha logrado cambios regresa al entorno.

\section{Referencias bibliográficas}

- Arocena, J. (2001). Una investigación de procesos de desarrollo local. En El desarrollo local: Un desafío contemporáneo. (pp. 201-229). Uruguay: Santillana.

- Artiga Gónzalez, A. (1993). Cuando la historia la hacen las mujeres: El caso de las comunidades de repatriados. Revista de Ciencias Sociales y Humanidades (32), pp. 131-152.

- Bertalanffy, L. (1987). Teoría general de los sistemas: Fundamentos, desar- 
rollo, aplicaciones. México: Fondo de Cultura Económica.

- Belkis, C. (2007). La ciudad democrática como ámbito, sistema y poder de integración social y cultural: Nuevos desafíos para los gobiernos locales. (Spanish). Provincia (18), pp. 81-95.

- Boisier, S. (2011). El territorio en la contemporaneidad (la recuperación de las políticas territoriales). Revista LIDER (19), pp. 9-24.

$\bullet$ . (2005). ¿Hay espacio para el desarrollo local en la globalización? Revista de la CEPAL (86), pp. 47-62.

- Boulding, K. E. (1956). General systems theorythe skeleton of science. Management Science (Pre-1986), 2(3), pp. 197.

- Comisión Mundial del Medio Ambiente y del Desarrollo. (1992). Nuestro futuro común. Madrid: Alianza Editorial.

- Cuenca Cabeza, M. (2000). Ocio humanista: Dimensiones y manifestaciones actuales del ocio. Bilbao: Universidad de Deusto.

- Easton, D. (1969). Esquema para el análisis político. Buenos Aires: Amorrortu.

- Fernández Cavia,J. (2013). Marcas de destino y evaluación de sitios web: Una metodología de investigación. (S.E.)

- Fernández Cavia, J. \& Huertas, A. (2014). La gestión de las marcas de destino y de territorio desde la perspectiva de las relaciones públicas. Comunicació: Revista De Recerca i d'Anàlisi, pp. 9-26

- Hawkes,J.(2001). The fourth pillar of sustainability: Culture's essential role in public planning. Melbourne, Vic: (at): Cultural Development Network.

- Johansen, O. (1993). Introducción a la teoría general de sistemas. Editorial Limusa.

- La Prensa Gráfica. (2014). Perquín celebrará la edición 22 del festival de invierno. Recuperado de: http://www.laprensagrafica.com/2014/07/30/ perquin-celebrara-la-edicion-22-del-festival-de-invierno

- Luhmann, N. (1998). Sistemas sociales: Lineamientos para una teoría general. Anthropos.

- Martín Baró, I. (1995). Acción e ideología. San Salvador: UCA Editores.

- Martín Baró, I., Blanco Abarca, A., \& Chomsky, N. (1998). Psicología de la liberación. Madrid: Trotta.

- Perquín. (2015). Página web del territorio de perquín. Recuperado de: http:// perquin.com.sv/ 
- Quartesan, A., Romis, M., \& Lanzafame, F. (2007). Las Industrias Culturales en América Latina y El Caribe: Desafíos y oportunidades. (S. E.)

- Ritchie, B. W. (1998). Bicycle tourism in the south island of New Zealand: Planning and management issues. Tourism Management 19(6), pp. 567-582

- Tobey, R. (2007). El encuentro intercultural visto con un enfoque sistémico. ULÚA. Revista de historia, sociedad y cultura (10), pp. 157-187. Recuperado de: http://goo.gl/kJOYVE

- UNESCO. (2015). Página web de la UNESCO. Recuperado de: http://goo. gl/iOVug1

$\cdot$ . (2014). Culture for development indicator. Francia. Recuperado de: http://goo.gl/NL13Q8 . (2009). Marco de estadísticas generales de la UNESCO. Montreal, Quebec, Canadá: UNESCO-UIS.

. (1982). Conferencia Mundial sobre las políticas públicas culturales. Declaración De México Sobre Las Políticas Públicas Culturales. México D. F. Recuperado de: http://goo.gl/wTgQak

- United Nations. (2015). Desarrollo sostenible: Antecedentes. Recuperado de: http://goo.gl/iOVug1

- Vázquez Barquero, A. (2000). Desarrollo endógeno y globalización. EURE. Revista Latinoamericana De Estudios Urbano Regionales 26(79), pp. 47-65. Doi: 104067/S0250-71612000007900003

- Vergara, N. (2012). "Significación social”y “territorio": Aproximaciones epistemológicas. Líder: Revista Labor Interdisciplinaria De Desarrollo Regional (21), pp. 9-18.

\section{Notas}

1 El presente artículo fue presentado en el Congreso OcioGune 2015, gracias al apoyo financiero de la Universidad Centroamericana "José Simeón Cañas" de El Salvador y a la Cátedra UnescoSantander para la formación de Recursos Humanos de América Latina. Una versión anterior de este artículo forma parte del libro Efectos sociales del ocio y las industrias creativas y culturales: Una mirada desde diversos ámbitos, colectivos y contextos. Compiladores: Cuenca Amigo, J., Ahedo González, R. (Documentos de Estudios de Ocio, 56). Universidad de Deusto, 2016. 